\title{
CORRIGENDUM
}

\section{Resistant starch and arabinoxylan augment SCFA absorption, but affect postprandial glucose and insulin responses differently - CORRIGENDUM}

\author{
Anne Krog Ingerslev, Peter Kappel Theil, Mette Skou Hedemann, Helle Nygaard Lærke \\ and Knud Erik Bach Knudsen
}

(First published online 16 February 2015)

doi:10.1017/S0007114513004066, Published by Cambridge University Press, February 2014.

The $P$ value for the diet effect on net portal glucose flux (NPF) (Table 4 ) in the paper by Ingerslev et al. ${ }^{(1)}$ was incorrect. It should read: The NPF of glucose were $258 \mathrm{mmol} / \mathrm{h}$ on the WSD, $203 \mathrm{mmol} / \mathrm{h}$ on the RSD and $242 \mathrm{mmol} / \mathrm{h}$ on the AXD (sEM 21 ), and the corresponding $P$ values were $P_{\text {Diet }}=0 \cdot 20, P_{\text {Time }}<0.001$ and $P_{\text {Diet } \times \text { Time }}<0 \cdot 001$, respectively.

Table 4. Net portal flux of metabolites from 0 to $5 \mathrm{~h}$ after the first daily meal ${ }^{\star}$

(Least-squares (LS) means with their standard errors or $95 \%$ confidence intervals)

\begin{tabular}{|c|c|c|c|c|c|c|c|c|c|c|}
\hline & \multicolumn{2}{|c|}{ WSD } & \multicolumn{2}{|c|}{ RSD } & \multicolumn{2}{|c|}{ AXD } & \multirow[b]{2}{*}{ SEM } & \multicolumn{3}{|c|}{$P$} \\
\hline & LS means & $95 \% \mathrm{Cl}$ & LS means & $95 \% \mathrm{Cl}$ & LS means & $95 \% \mathrm{Cl}$ & & Diet & Time & Diet $\times$ time \\
\hline \multicolumn{10}{|l|}{ Net portal flux $(\mathrm{mmol} / \mathrm{h})$} & NS \\
\hline Glucose & 258 & & 203 & & 242 & & 21 & 0.20 & $<0.001$ & $<0.001$ \\
\hline Insulin flux $(\mathrm{nmol} / \mathrm{h}) \S$ & $9 \cdot 0^{\mathrm{a}, \mathrm{b}}$ & $3 \cdot 6,15 \cdot 7$ & $10 \cdot 5^{a}$ & $4 \cdot 8,17 \cdot 5$ & $4 \cdot 6^{\mathrm{b}}$ & $0 \cdot 01,10 \cdot 2$ & - & 0.09 & 0.04 & NS \\
\hline C-peptide $(\mathrm{nmol} / \mathrm{h}) \S$ & 7.9 & $4 \cdot 6,11 \cdot 4$ & $6 \cdot 6$ & $3 \cdot 4,10 \cdot 0$ & $5 \cdot 5$ & $2 \cdot 4,8 \cdot 8$ & - & NS & $<0.001$ & NS \\
\hline GLP-1 $(\mathrm{nmol} / \mathrm{h}) \S$ & 1.6 & $1 \cdot 2,2 \cdot 0$ & 1.8 & $1 \cdot 4,2 \cdot 2$ & 1.9 & $1 \cdot 4,2 \cdot 3$ & - & NS & $<0.001$ & NS \\
\hline GIP $(n m o l / h) \S$ & 4.4 & $2 \cdot 1,6 \cdot 9$ & $2 \cdot 8$ & $0.5,5 \cdot 2$ & $3 \cdot 3$ & $1 \cdot 1,5 \cdot 7$ & - & NS & 0.12 & NS \\
\hline SCFA & $37^{a}$ & & $66^{\mathrm{b}}$ & & $102^{\mathrm{C}}$ & & 9.3 & $<0.001$ & 0.009 & 0.006 \\
\hline Acetate & $24^{\mathrm{a}}$ & & $40^{\mathrm{b}}$ & & $62^{c}$ & & $6 \cdot 1$ & $<0.001$ & 0.006 & 0.01 \\
\hline Propionate & $8 \cdot 2^{\mathrm{a}}$ & & $17^{\mathrm{b}}$ & & $25^{\mathrm{c}}$ & & $2 \cdot 6$ & 0.001 & 0.03 & 0.002 \\
\hline Butyrate & $2 \cdot 8^{a}$ & & $5 \cdot 7^{\mathrm{a}}$ & & $10 \cdot 2^{\mathrm{b}}$ & & 1.0 & 0.001 & 0.02 & 0.04 \\
\hline Valerate & $1 \cdot 7^{\mathrm{a}}$ & & $2 \cdot 4^{\mathrm{b}}$ & & $2 \cdot 5^{\mathrm{b}}$ & & 0.4 & 0.05 & $<0.001$ & 0.001 \\
\hline Caproate & $0.05^{\mathrm{a}}$ & & $0.68^{\mathrm{b}}$ & & $0.59^{b}$ & & 0.15 & 0.03 & 0.005 & 0.03 \\
\hline BCFA & $1 \cdot 3^{\mathrm{a}}$ & & $1 \cdot 6^{\mathrm{a}}$ & & $2 \cdot 8^{\mathrm{b}}$ & & 0.26 & $<0.001$ & NS & 0.08 \\
\hline Isobutyrate & $0.6^{\mathrm{a}}$ & & $0.7^{\mathrm{a}}$ & & $1 \cdot 2^{\mathrm{b}}$ & & 0.11 & $<0.001$ & NS & 0.09 \\
\hline Isovalerate & $0.7^{\mathrm{a}}$ & & $0.9^{a}$ & & $1.5^{\mathrm{b}}$ & & 0.15 & $<0.001$ & 0.08 & 0.07 \\
\hline \multicolumn{11}{|l|}{ Hepatic extraction (\%) } \\
\hline Glucose & 8 & & 5 & & 9 & & 4.2 & NS & $<0.001$ & NS \\
\hline Insulin & 27 & & 44 & & 44 & & 11 & NS & NS & NS \\
\hline Total SCFA & 57 & & 50 & & 63 & & $9 \cdot 2$ & NS & 0.03 & NS \\
\hline Acetate & $44^{a, b}$ & & $29^{b}$ & & $49^{a}$ & & 12 & 0.03 & NS & NS \\
\hline Propionate & 94 & & 93 & & 95 & & 1.4 & NS & NS & NS \\
\hline Butyrate & 83 & & 79 & & 83 & & $4 \cdot 6$ & NS & $<0.001$ & NS \\
\hline
\end{tabular}

WSD, Western-style diet; RSD, resistant starch-enriched diet; AXD, arabinoxylan-enriched diet; GLP-1, glucagon-like peptide 1; GIP, glucose-dependent insulinotropic peptide; BCFA, branched-chain fatty acids.

a,b,c Mean values within a row with unlike superscript letters are significantly different $(P<0.05)$.

${ }^{*}$ Mean plasma concentrations were determined in pigs fed either WSD, RSD or AXD.

$\dagger$ Mean values for portal blood flow are determined based on initial blood flow measurements.

$\ddagger P>0 \cdot 1$.

$\S$ Since data were logarithmically transformed before data analysis, SEM is not the correct measure of variance. Instead, $95 \% \mathrm{Cl}$ are given 
In the abstract, the first sentence describing the results (page 1564, line 7) should be: 'The NPF of insulin was lower $(P=0 \cdot 04)$ in AXD-fed pigs $(4.6 \mathrm{nmol} / \mathrm{h})$ compared with RSD-fed pigs $(10.5 \mathrm{nmol} / \mathrm{h})$, despite similar glucose absorption among diets $\left(203-258 \mathrm{mmol} / \mathrm{h}, P_{\text {Diet }}=0 \cdot 20\right)$. A prolonged and a lower peak NPF of glucose was observed in RSD-fed pigs $\left(P_{\text {Diet } \times \text { Time }}<0 \cdot 001\right)$ compared with the WSD- and AXD-fed pigs.'

In the Results section, first paragraph (page 1570, line 9), the sentence should be: 'Substituting wheat flour with potato and high-amylose maize starches or rye flakes and enzyme-treated wheat bran did not affect the NPF of glucose $(P=0 \cdot 20)$. However, RSD-fed pigs showed a significant diet $\times$ time interaction $(P<0 \cdot 001)$; RSD-fed pigs had a lower peak NPF of glucose (approximately $300 \mathrm{mmol} / \mathrm{h}$ ) between 45 and $120 \mathrm{~min}$ after feeding.'

The authors apologise for this error.

\section{Reference}

1. Ingerslev AK, Theil PK, Hedemann MS, et al. (2014) Resistant starch and arabinoxylan augment SCFA absorption, but affect postprandial glucose and insulin responses differently. Br J Nutr 111, 1564-1576. Published by Cambridge University Press, February 2014, doi:10.1017/S0007114513004066. 